\title{
The Role of Cavity Losses on Non-Adiabatic Couplings and Dynamics in Polaritonic Chemistry
}

\author{
Panayiota Antoniou, Figen Suchanek, James F. Varner, and Jonathan J. Foley \\ IV* \\ Department of Chemistry, William Paterson University, 300 Pompton Road, Wayne, NJ, \\ 07470, USA \\ E-mail: foleyj10@wpunj.edu
}

\begin{abstract}
We present a non-Hermitian formulation of the polaritonic structure of azobenzene strongly coupled to a photonic mode that explicitly accounts for the fleeting nature of the photonmolecule interaction. This formalism reveals that the polaritonic non-adiabatic couplings that facilitate cis-trans isomerization can be dramatically modified by photonic dissipation. We perform Fewest-Switches Surface Hopping dynamics on the surfaces that derive from our nonHermitian formalism and find that the polaritonic isomerization yields are strongly suppressed for moderate dissipation rates, and that cavity-free isomerization dynamics are recovered under large dissipation rates. These findings highlight the important role that the finite lifetime of photonic degrees of freedom play in polaritonic chemistry.
\end{abstract}

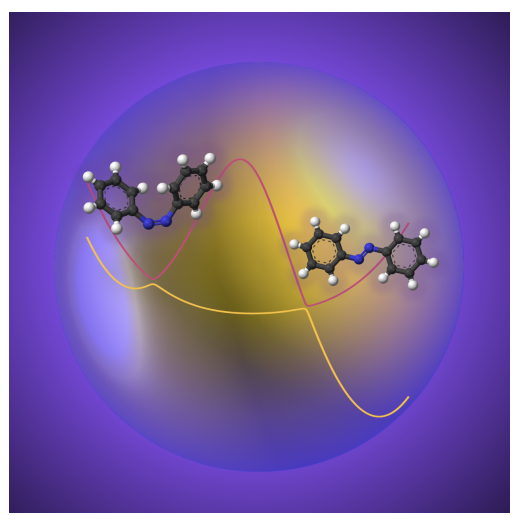

Molecular polaritons arise when the interaction between light and molecular transitions is large compared to the dissipative energy scales in the system. ${ }^{1-9}$ Strong coupling to photons can be realized by placing the molecular system in an optical cavity tuned to be resonant with a molecular transition, ${ }^{2,4,8}$ or in the vicinity of nanomaterials that support strong optical modes, including plasmon resonances ${ }^{1,6}$ or scattering-mediated absorption resonances. ${ }^{10}$ Ebbesen and co-workers demon- strated experimentally that the reactivity of polaritonic systems can be dramatically different than that of the lone molecular system. ${ }^{2,5}$ Subsequently, a wealth of theoretical studies have suggested that strong coupling can reshape the energy landscape that dictates energy transfer and reactivity. ${ }^{4,7,8,11-23}$

Azobenzene has been studied as a prototypical molecule for photoisomerization that can be potentially used as a molecular photo-switch or energy storage material, among other applications. ${ }^{24,25}$ The excited-state dynamics of azobenzene accessible by visible-light excitation are guided by a quasi-harmonic potential along the isomerization coordinate $(R)$ with a minimum that approximately coincides with the transition-state value of $R$ on the ground-state surface. Hence, photochemical activation of cisazobenzene yields the trans- isomer with nearly equal likelihood as relaxation back to the cisisomer $^{8,13}$ (see Figure 1). 
Unlike traditional photochemistry where the interaction between light and molecules is perturbative, polaritonic chemistry occurs when light hybridizes with molecular degrees of freedom, causing the potential energy surfaces to inherit properties of the photonic and molecular systems. For example, polaritonic potential energy surfaces may develop new critical points, new intersections or avoided crossings, and different couplings between surfaces; these new features may suppress or enhance the reactivity compared to the lone molecular system. Azobenzene strongly coupled to a photon can illustrate all of these features depending on the details of the photon and its confinement ${ }^{4,7,8,17,20}$ (see Figure 1).

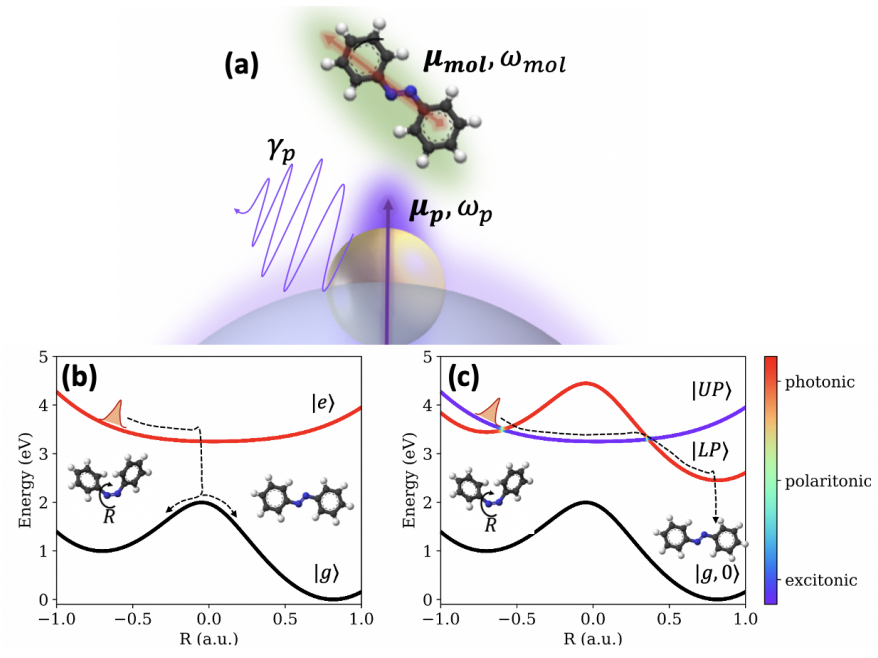

Figure 1: (a) Schematic of azobenzene coupled through its electronic transition dipole moment $\left(\mu_{m o l}\right)$ to the effective transition dipole moment of a localized photonic resonance in an optical nanostructure $\left(\mu_{p}\right)$. The frequency of the molecular transition $\left(\omega_{m o l}\right)$, the frequency of the photonic resonance $\left(\omega_{p}\right)$, and the dissipation rate of the photonic resonance $\left(\gamma_{p}\right)$ all impact the polaritonic structure. (b) The singlet ground- $(|g\rangle)$ and first excited-state $(|e\rangle)$ potential energy surfaces of azobenzene along the reaction coordinate $R$. (c) The composite singlet ground, zero-photon state $(|g, 0\rangle)$, lower polariton $(|L P\rangle)$ and upper polariton $(|U P\rangle)$ potential energy surfaces along $R$ that result when $\hbar \omega_{m o l}=\hbar \omega_{p}=2.45 \mathrm{eV}$ and $\hbar g=0.02 \mathrm{eV}$, where $\hbar g \propto \mu_{m o l} \cdot \mu_{p}$.

Polaritonic chemistry is an inherently multiscale problem that has motivated considerable theoretical and computational effort. In the case of electronic strong coupling where light is strongly interacting with molecular electronic transitions (e.g. the $S_{0}$ to $S_{1}$ transition of azobenzene considered in this work), the photonic and molecular electronic degrees of freedom are often treated on the same quantum mechanical footing, while the nuclear degrees of freedom may be treated as slow or classical degrees of freedom. ${ }^{4,12,14,21}$ Drawing on this Cavity Born-Oppenheimer approximation, the nuclear dynamics in polaritonic chemistry have been simulated classically using Ehrenfest dynamics ${ }^{19}$ and Fewest-Switches Surface Hopping (FSSH), ${ }^{17,19-21}$ and quantum mechanically using the framework of exact factorization, ${ }^{7}$ the partial linearized density matrix path-integral approach, ${ }^{8}$ wavepacket propagation, ${ }^{22}$ and the multi-configuration timedependent Hartree approach. ${ }^{23}$ Interestingly, a number of semiclassical dynamics methods have been studied in the context of atomic polaritonic systems that treated the atomic electronic degrees of freedom quantum mechanically, and the photonic degrees of freedom classically. ${ }^{26}$ In that work, it was found that FSSH dynamics did not recover important interference effects that are important, for example, in polaritonic emission phenomena. ${ }^{26}$ However, because of the large effective nuclear mass associated with the reactive degree of freedom we are simulating in this work (see Table S2), we do not expect such a deficiency will be significant for our results. We note that polaritonic chemistry can also arise from vibrational strong coupling, where light is strongly interacting with vibrational transitions, ${ }^{5}$ though this is not the focus of the current investigation. In electronic strong coupling, molecular electronic and photonic degrees of freedom have been treated using model and/or semi-empirical Hamiltonians, ${ }^{8,11,13,15,17}$ although there has been a recent surge in activity focused on merging $a b$ initio molecular electronic structure theory with cavity quantum electrodynamics ( $a b$ initio CQED) to provide an accurate and predictive model of polaritonic structure. ${ }^{12,27-32}$ Here we couple a non-Hermitian CQED Hamiltonian ${ }^{22,23,33}$ to a model Hamiltonian for the molecular electronic structure of azobenzene to simulate the polaritonic structure and dynamics with ex- 
plicit inclusion of finite cavity lifetimes. We utilize FSSH dynamics ${ }^{17,20,21,34-37}$ with potential energy surfaces and non-adiabatic couplings from our non-Hermitian polaritonic structure theory to elucidate the impact of cavity lifetime on the isomerization dynamics under other cavity parameters previously found to facilitate facile cis-to-trans isomerization. ${ }^{8,17} \mathrm{Sev}$ eral independent contemporaneous studies have leveraged non-Hermitian Hamiltonians to investigate cavity losses in polaritonic chemistry. Feist and co-workers ${ }^{22}$ employed full quantum simulations of the photorelaxation dynamics of uracil, and found conditions whereby dissipation and coupling strength conspire to optimize photoprotection. ${ }^{22}$ Ulusoy and Vendrell utilized the multiconfiguration time-dependent Hartree approach with non-Hermitian Hamiltonians to study the polariton dynamics of $\mathrm{NaI}$ and pyrazine in both the single-molecule and ensemble limit for a variety of cavity dissipation rates. ${ }^{23}$ We note that since the light-matter coupling strength scales with the square root of the number of molecules coupled to the cavity, such ensembles of molecules are often required to experimentally realize strong coupling. ${ }^{23} \mathrm{Ad}-$ ditionally, Kossoski and Barbatti independently developed and rigorously validated a complexsurface FSSH method and demonstrated its applicability in the context of dissociative electron attachment dynamics. ${ }^{37} \mathrm{~A}$ important contribution of our non-Hermitian approach is the elucidation of how cavity losses impact upon the polaritonic structure itself, which then impacts the dynamics that occur on one or more polaritonic surfaces. In the model azobenzene system we explore, we find three distinct regimes with respect to the cavity dissipation rate: low dissipation rates $\left(\hbar \gamma<10^{-0.5} \mathrm{meV}\right.$, or $\tau \equiv$ $1 / \gamma>2 \mathrm{ps})$ where polaritonic isomerization to the trans configuration is facile, moderate dissipation rates $\left(10^{0.5}<\hbar \gamma \leq 10^{1.5} \mathrm{meV}\right.$, or $2 \cdot 10^{-1}>\tau \geq 2 \cdot 10^{-2} p s$ ) where isomerization to the trans configuration is strongly suppressed, and large dissipation rates $\left(\hbar \gamma \geq 10^{2}\right.$ meV or $\tau \leq 10^{-2.5}$ ps where the isomerization yield tends to nearly equal likelihood for cis and trans configurations similar to the photoisomerization of lone azobenzene (see Figure S1 in the Supporting Information). We find that the couplings between the polaritonic surfaces are quantitatively altered by the cavity dissipation rates, and that the dissipative features of the photon are mixed into the polaritonic surfaces in regions of strong non-adiabaticity.

Following previous studies, ${ }^{8,17}$ we use a generalized Rabi Hamiltonian for a single azobenzene molecule coupled to a single photonic mode:

$$
\hat{H}_{T o t}=\hat{H}_{e l}+\hat{H}_{p}+\hat{H}_{e l, p}
$$

where $\hat{H}_{e l}$ denotes the electronic Hamiltonian:

$$
\hat{H}_{e l}=E_{g}(R) \hat{a}_{g}^{\dagger} \hat{a}_{g}+E_{e}(R) \hat{a}_{e}^{\dagger} \hat{a}_{e},
$$

where $E_{g}(R) \quad\left(E_{e}(R)\right)$ denotes the ground(excited-) state electronic energy at nuclear coordinate $R$, and $\hat{a}_{p}^{\dagger}\left(\hat{a}_{p}\right)$ are the creation (annihilation) operators for electronic state $p$. The nuclear coordinate $R$ here denotes the dihedral angle of the Ph-N-N-Ph system that defines the cis-trans isomerization (see Figure 1). The electronic states as a function of the nuclear coordinate are parameterized following the work of Mandal and $\mathrm{Huo}^{8}$ (see Supporting Information for more details).

The cavity resonances have finite lifetimes due to their coupling to a continuum of states outside the cavity; attributing a complex frequency to the photonic degrees of freedom provides a simple way to model this finite lifetime without explicit inclusion of additional continuum degrees of freedom. ${ }^{23,38}$ Additionally as we will show, the resulting non-Hermitian formulation imparts the polaritonic structure, i.e. the potential energy surfaces and non-adiabatic couplings, with these dissipative features through imaginary contributions to these quantities. We write the photonic Hamiltonian as

$$
\hat{H}_{p}=\hbar\left(\omega-i \frac{\gamma}{2}\right) \hat{b}^{\dagger} \hat{b}
$$

where $\hat{b}^{\dagger}(\hat{b})$ denote the raising (lowering) operator for the photon state with complex frequency $\tilde{\omega}=\omega-i \frac{\gamma}{2}$, where $\gamma$ arises from the finite-lifetime of the photonic resonance. We parameterize the photonic Hamiltonian to have $\hbar \omega=2.45 \mathrm{eV}$ and $\hbar \gamma$ to have values in the range 
0.01 to $1000 \mathrm{meV}$ (equivalently, cavity lifetimes in the range 0.0006 to $63 \mathrm{ps}$ ). Such a nonHermitian form of this photonic Hamiltonian was recently considered by Cortes, Otten, and Gray $^{33}$ and captures important dissipative effects if the photonic degrees of freedom. Finally, the interaction Hamiltonian has the form ${ }^{8,17,33}$

$$
\hat{H}_{e l, p}=\hbar g\left(\hat{b}^{\dagger}+\hat{b}\right)\left(\hat{a}_{e}^{\dagger} \hat{a}_{g}+\hat{a}_{g}^{\dagger} \hat{a}_{e}\right) ;
$$

in this work, we neglect the dipole self-energy term in the polaritonic Hamiltonian, which has recently been found to play a key role in the polaritonic structure and dynamics of systems with multiple photonic modes ${ }^{39}$ and/or systems in the ultra-strong coupling regime. ${ }^{40}$ Neglect of the dipole self energy, as well as truncation of the electronic basis, breaks the gauge invariance of our Hamiltonian. We refer interested readers to several insightful discussions of gauge invariance and gauge ambiguities that arise from term and/or basis truncation in dipole gauge Hamiltonians (such as the one used here) after gauge transformation from a minimal coupling Hamiltonian. ${ }^{41-43}$

Here we consider a 1-photon 1-molecule system in which the coupling term has the value $\hbar g=0.02 \mathrm{eV}$ (i.e. not in the ultra-strong coupling regime); in recent work by Mandal and Huo, this coupling along with a photon energy of $2.45 \mathrm{eV}$ was shown to be particularly facile for polaritonic isomerization. ${ }^{8}$ We can express the Hamiltonian matrices in a local basis that includes states where energy is localized as a photon in the cavity mode, in the molecular system as an exciton, in both, or in neither. In other words, this system permits the following four composite basis states $\left|\phi_{i}\right\rangle \in$ $\{|g, 0\rangle,|g, 1\rangle,|e, 0\rangle,|e, 1\rangle\}$, where the composite state $\left|\phi_{1}\right\rangle=|g, 0\rangle$ denotes the molecule is in its ground state and no photon in the cavity. The various contributions to the Hamiltonian matrices are shown in the Supporting Information. The new polaritonic potential energy surface can be identified by building the total Hamiltonian matrix in this basis, $\mathbf{H}_{T o t}$, at different values of $R$, diagonalizing the matrix, and identifying the eigenvalues $\epsilon_{\alpha}(R)$; similarly, the energy eigenstates in the polariton basis, $\left|\Phi_{\alpha}(R)\right\rangle$, will be the eigenvectors of this matrix. Diagonalizing the non-Hermitian $\mathbf{H}_{\text {Tot }}$ yields left and right eigenvectors. The left eigenvectors are complex conjugates of the right eigenvectors (see Figure S2 in the Supporting Information); we use the basis of right eigenvectors as the polariton basis in this work. At geometries where $E_{g}(R)+\hbar \tilde{\omega} \approx E_{e}(R)$, we get strong mixing of the $|g, 1\rangle$ and $|e, 0\rangle$ states. That is, $\left|\Phi_{2}\right\rangle$ and $\left|\Phi_{3}\right\rangle$ will be linear combinations of the form

$$
\left|\Phi_{\alpha}(R)\right\rangle=\sum_{i} c_{i}^{\alpha}(R)\left|\phi_{i}\right\rangle
$$

that will typically have strong contributions coming from basis states $\left|\phi_{i}\right\rangle \in\{|g, 1\rangle,|e, 0\rangle\}$ at values of $R$ where the photon is resonant with the excitonic transition energy. We also refer to states $\left|\Phi_{2}\right\rangle$ and $\left|\Phi_{3}\right\rangle$ as $|L P\rangle$ and $|U P\rangle$, respectively, to denote their polaritonic character (see Figure 1). These polariton states will have associated potential energy surfaces defined by $\epsilon_{2}(R)$ and $\epsilon_{3}(R)$ that may differ qualitatively from the uncoupled surfaces, potentially imparting different reactivity to the system. Similar to previous studies, ${ }^{8,13}$ we can also elucidate the nature of the polaritonic surfaces as a function of geometry; i.e. some regions of the surface will take on a strong photonic character (when $\left|c_{2}^{\alpha}(R)\right|^{2} \approx 1$ ), some will take on a strong excitonic character $\left(\left|c_{3}^{\alpha}(R)\right|^{2} \approx 1\right)$, and others a mixed or polaritonic character $\left(\left|c_{2}^{\alpha}(R)\right|^{2} \approx\right.$ $\left.\left|c_{3}^{\alpha}(R)\right|^{2} \approx 0.5\right)$. That said, for all geometries, $\left|\Phi_{1}(R)\right\rangle \approx|g, 0\rangle$ and $\left|\Phi_{4}(R)\right\rangle \approx|e, 1\rangle$ with eigenvalues $\epsilon_{1}(R) \approx E_{g}(R)$ and $\epsilon_{4}(R) \approx$ $E_{e}(R)+\hbar \omega$. We also note that the polariton basis is not necessarily orthogonal in this nonHermitian formulation. In particular, the $|L P\rangle$ and $|U P\rangle$ have non-zero overlap at geometries where there strong polaritonic character (see Figure S3 in the Supporting Information).

We track the nuclear motion along a single reactive coordinate $R$ using FSSH dynamics; ${ }^{17,20,21,34-37}$ details of this approach are provided in the Supporting Information.

The polaritonic degrees of freedom are represented quantum mechanically by a wavefunction that can be written as a superposition of polariton eigenstates, $|\Psi(R, t)\rangle=$ 
$\sum_{\alpha} C_{\alpha}(t)\left|\Phi_{\alpha}(R)\right\rangle$. The time-dependence of these coefficients obeys the time-dependent Schrödinger equation,

$$
\dot{\mathbf{C}}(t)=-\frac{i}{\hbar} \mathbf{H}_{P l} \cdot \mathbf{C}(t)-\dot{R} \mathbf{d} \cdot \mathbf{C}(t),
$$

where $\mathbf{d}$ denotes the derivative coupling matrix, ${ }^{35}$ and $\mathbf{H}_{P l}$ is the non-Hermitian polariton Hamiltonian matrix; the dependence on the nuclear coordinate for both of these matrices is implied. The elements of the derivative coupling matrix are defined as $d_{\alpha, \beta}(R)=$ $\left\langle\Phi_{\alpha}(R) \mid \frac{d}{d R} \Phi_{\beta}(R)\right\rangle$, where forward finite differences are used to compute the derivatives of the polaritonic eigenfunctions. We note that this derivative coupling term is often computed with a perturbative expression, $d_{\alpha, \beta}(R)=$ $\frac{\left\langle\Phi_{\alpha}\left|\hat{H}_{P l}^{\prime}\right| \Phi_{\beta}\right\rangle}{E_{\beta}-E_{\alpha}}$, but this expression assumes an orthogonal basis, which is not necessarily the cases with our polariton basis (see Figure S5 in the Supporting Information).

Although the polaritonic wavefunction is typically in a superposition of multiple polaritonic eigenfunctions, in the FSSH scheme, the nuclei feel a force from a single polaritonic surface at a time, but can transition or "hop" between surfaces with a probability that is updated at each time-step. We compute the probability for hopping from surface $\alpha$ to $\beta$ to be given by $P_{\alpha, \beta}(t)=\max \left(\frac{\dot{\rho}_{\beta}(t) d t}{\rho_{\alpha}(t)}, 0\right)$, where $\dot{\rho}_{\beta}(t)$ is the time-derivative of the population of the polaritonic state $\Phi_{\beta}$ at time $t$ and $\rho_{\alpha}(t)$ is the population of polaritonic state $\Phi_{\alpha}$ at time $t$, defined as $\rho_{\alpha}(t)=C_{\alpha}^{*}(t) C_{\alpha}(t)$. Propagation of Eq. 6 with a complex $\mathbf{H}_{P l}$ does not conserve the norm of $\mathbf{C}$; rather, the norm of the polaritonic wavefunction decays with time. This models the radiative decay of the polaritonic system (i.e. loss of a photon to the continuum of states outside the cavity) mediated exclusively by the cavity through transitions from the $|g, 1\rangle$ contribution to the $|U P\rangle$ and $|L P\rangle$ states to state $|g, 0\rangle$. As a consequence, we equate loss of norm in a given timestep to relaxation to the ground state $\left(\left|\Phi_{1}\right\rangle \approx|g, 0\rangle\right)$, which allows us to compute the instantaneous timederivative of the ground state population as $\dot{\rho}_{1}(t)=-\dot{\rho}_{3}(t)-\dot{\rho}_{2}(t)$. The spontaneous emis- sion rate of the molecules could also be modeled using complex frequencies, but we choose to neglect this effect since this rate is typically slower than the cavity dissipation rate and has a strong dependence of the geometry along the reaction coordinate $R .{ }^{44}$ At each time-step, as uniform random number $\xi \in[0,1]$ is chosen, and a surface hop to state $\beta<\alpha$ is performed when $\sum_{i=1}^{\beta-1} P_{\alpha, i}<\xi \leq \sum_{i=1}^{\beta} P_{\alpha, i}$.

In Figure 2, the polaritonic surfaces are shown when $\hbar \gamma=10 \mathrm{meV}$ (panel (a) and (c), and $\hbar \gamma=100 \mathrm{meV}$ (panel (b) and (d)). Two qualitative features of the surfaces are altered by the magnitude of $\gamma$ : the avoided crossing between the surfaces under low loss and moderate loss conditions becomes an intersection with high loss, and the polaritonic character near the crossing feature is strongly diminished in the high loss case (see Figure 2). The energy
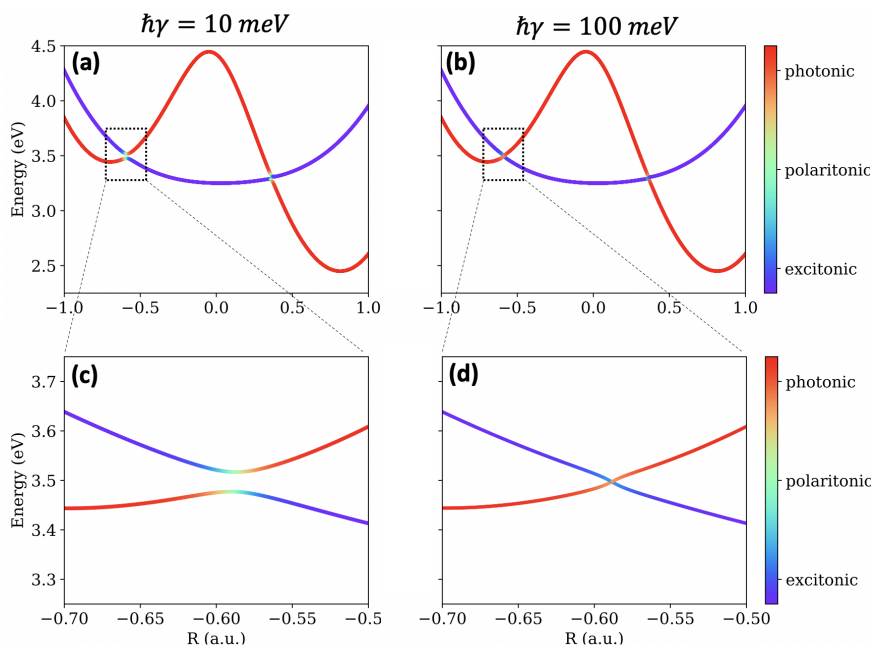

Figure 2: Polaritonic potential energy surfaces when $\hbar \gamma=10 \mathrm{meV}$ (a) and (c), and when $\hbar \gamma=100 \mathrm{meV}$ (b) and (d)

gap of the avoided crossing at the geometry $R \approx-0.6$, where the molecular excitation energy is in resonance with the cavity, can be understood in terms of energy eigenvalues of the Jaynes-Cummings ${ }^{45}$ model,

$$
\frac{E_{ \pm}}{\hbar}=\omega-i \frac{\gamma}{4} \pm \sqrt{g^{2}-\frac{\gamma^{2}}{16}}
$$

where $\omega$ is the frequency of the molecular transition and the cavity photon, and $g$ and $\gamma$ quantify the coupling strength and cavity dissipation rate as before. The difference between $E_{+}$ 
(energy of the $|U P\rangle$ state) and the $E_{-}$(energy of the $|L P\rangle$ state) is known as the Rabi splitting, which approaches zero as $\gamma$ approaches $4 g$ (see Figure 3 a for a map of absolute magnitude of the Rabi splitting values for variety of different $g$ and $\gamma$ values). The vanishing of the avoided crossing for large values of $\gamma$ (larger than $80 \mathrm{meV}$ or $0.08 \mathrm{eV}$ to be precise, since $\hbar g=0.02 \mathrm{eV}$ ) as shown in Figure 2 reflects the real part of the Rabi splitting going to zero. When $\gamma>4 g$, the Rabi splitting is imaginary (see the lower diagonal of the map in Figure 3 a. The $\gamma$ dependence on the $|U P\rangle$ and $|L P\rangle$ surfaces and their splitting has important consequences for the derivative couplings that drive non-adiabatic transitions in the nuclear dynamics, and for dissipative behavior that leads to transitions to the $|g, 0\rangle$ state. The leading term in the perturbative approximation to the derivative coupling between $|U P\rangle$ and $|L P\rangle$ is inversely proportional to the energy gap between these states; thus the magnitude of the derivative coupling increases as the splitting approaches zero. We compute the absolute magnitude derivative coupling between the upper-polariton and lower-polariton surfaces (i.e. $\left|d_{3,2}\right|$ or $\left|d_{U P, L P}\right|$ ) for the same range of $g$ and $\gamma$ values as were used to evaluate the Rabi splitting. The maximum value of this coupling in the vicinity of the crossing ( $R \approx-0.6$ a.u.) is plotted for each value of $g$ and $\gamma$, showing that $\left|d_{U P, L P}\right|$ is maximized when $\gamma=4 g$, and approaches zero for $\gamma>4 g$ (see Figure $3 \mathbf{b}$ ). As a consequence, for large loss values, one would expect the dynamics on the $|U P\rangle$ surface to follow typical adiabatic dynamics reminiscent of the excited-state dynamics of lone azobenzene. That is, with large loss values, the $|U P\rangle$ surface experiences negligible coupling to the $|L P\rangle$ surface and retains the curvature of the $|e, 0\rangle$ surface (see Figure S1). Also in this high-loss limit, the Rabi splitting is imaginary which results in avoided crossings of the imaginary component of the $|L P\rangle$ and $|U P\rangle$ surfaces. These imaginary components are represented for high- $(\hbar \gamma=100 \mathrm{meV})$, moderate$(\hbar \gamma=10 \mathrm{meV})$, and low loss $(\hbar \gamma=0.1 \mathrm{meV})$ cases in Figure 4, where imaginary part of the $|L P\rangle$ surfaces are plotted with dashed lines and
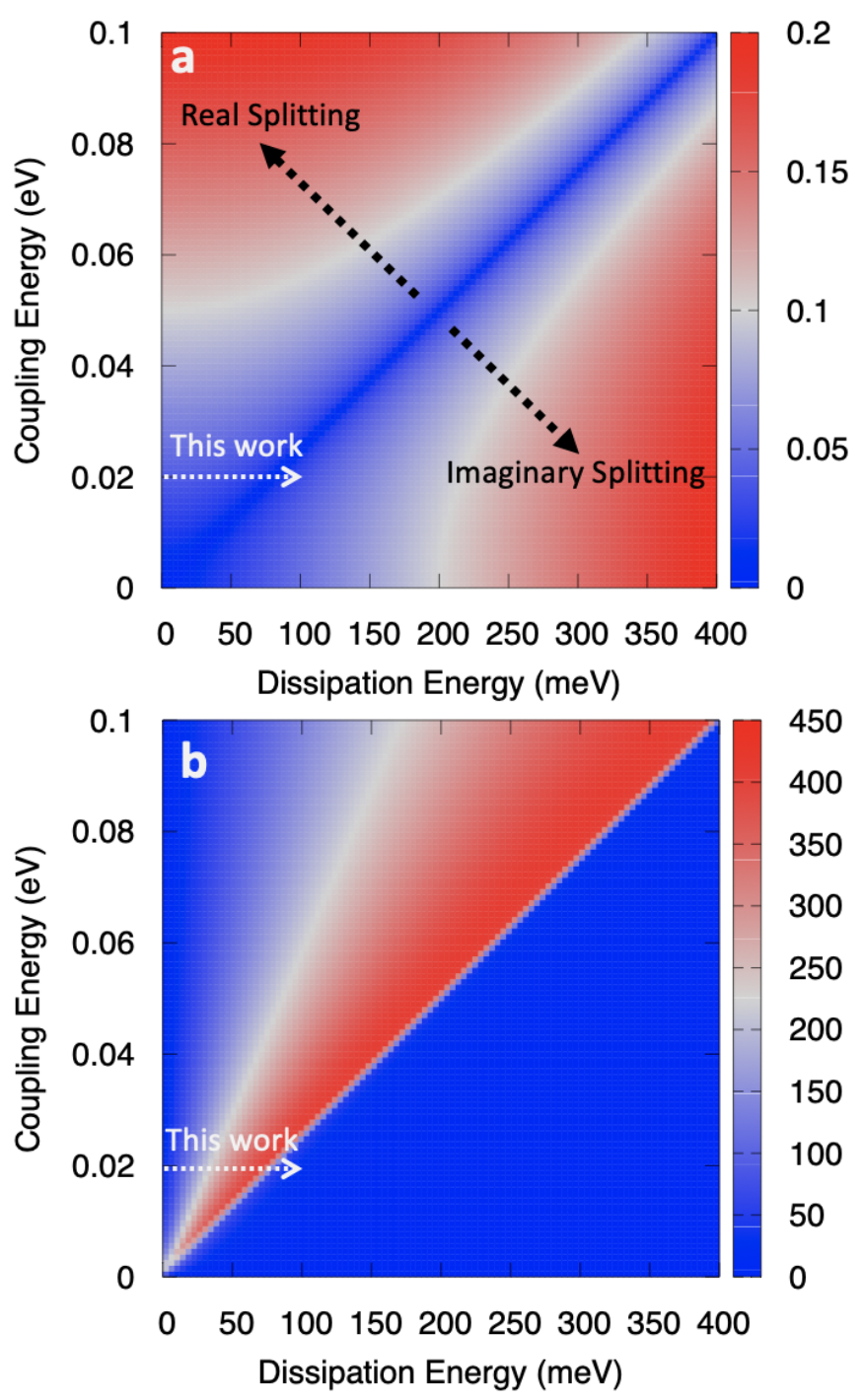

Figure 3: a Rabi Splitting from the Jaynes-Cummings model as a function of $\hbar \gamma$ and $\hbar g$, showing that the splitting goes to zero when $\gamma=4 g$. The Rabi splitting is real for values of $\gamma<4 g$, and imaginary for $\gamma>4 g$. b The maximum magnitude of the derivative coupling between the $|U P\rangle$ and $|L P\rangle$ state in the vicinity of their crossing ( $R \approx-0.6$ a.u. $)$, showing that this coupling approaches a maximum as the Rabi splitting approaches zero, whereas the magnitude of this coupling goes to zero when $\gamma>4 g$. The white dashed arrows are used to highlight the range of $\gamma$ and $g$ simulated by FSSH in this work.

the imaginary part of the $|U P\rangle$ surface are plotted with solid lines. An interesting consequence of the imaginary splitting in the high loss case is that the $|L P\rangle$ state inherits the majority of the cavity loss, whereas the dissipative nature of $|U P\rangle$ is much less significant (see Figure 4). Because the Rabi splitting is pure 
real when $\gamma<4 g$, we observe crossings between the imaginary parts of the $|L P\rangle$ and $|U P\rangle$ surfaces around $R \approx-0.6$ a.u. where nonadiabatic transitions tend to occur in the lowand moderate-loss cases (see Figure 4). This suggests the potential for dissipation to $|g, 0\rangle$ to compete with isomerization in the regime of moderate cavity losses where the non-adiabatic couplings are still large and both the $|U P\rangle$ and $|L P\rangle$ surfaces have dissipative character from their photonic contributions.

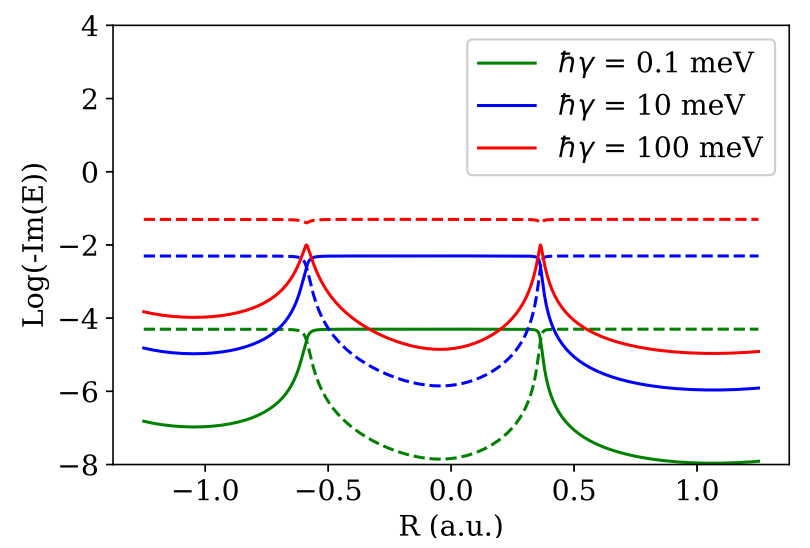

Figure 4: Imaginary part of the potential energy surface of the $|U P\rangle$ (solid lines) and $|L P\rangle$ (dashed lines) states for three different cavity dissipation rates; a logarithmic scale is used to aid visual comparison across several decades of loss. We see that for the low and moderate loss rates, the $|U P\rangle$ and $|L P\rangle$ states cross in the imaginary energy space, indicating that both states inherit the dissipative features of the cavity at certain geometries. For high losses, the upper and lower polariton surfaces are split in imaginary energy space, indicating that it is primarily the $|L P\rangle$ surfaces that inherits the dissipative features of the cavity in this regime.

The inferences made based on analysis of the features of the $\gamma$-dependent polaritonic structure are confirmed by FSSH dynamics ${ }^{20,21,34-37}$ simulating the polaritonic isomerization across a range of cavity loss values between 0.01 and $1000 \mathrm{meV}$ (equivalently, cavity lifetimes between 0.0006 and 63 ps). Loss values of 100 $\mathrm{meV}$ are typical of plasmonic cavities, while the low-loss cases could be realized in dielectric micro-resonators. ${ }^{6,10,46-48}$ For each cavity loss value, we run $500 \mathrm{FSSH}$ trajectories starting in the cis configuration on the $|U P\rangle$ surface. The random distribution of initial positions and velocities for these trajectories are discussed in the Supporting Information. It is observed that cis-to-trans isomerization is very facile under low loss conditions, consistent with prior polaritonic studies on azobenzene that considered lossless cavities ${ }^{8}$ or treated the losses a posteriori $^{17}$ (see Figure 5). In high-loss conditions, the isomerization dynamics approaches a $50 \%$ - $50 \%$ yield reminiscent of the cavity-free isomerization under high-loss conditions, which is consistent with the findings reported by Ulusoy and Vendrell that the dynamics of polaritonic systems with sufficiently lossy cavities resembles the dynamics of cavity-free systems. ${ }^{23}$ There is a small but noticeable oscillation in the isomerization yield whereby the trans yield when $\hbar \gamma=316 \mathrm{meV}$ is smaller than at 100 and $1000 \mathrm{meV}$. We have confirmed this oscillation persists after averaging over an additional 500 FSSH trajectories, suggesting they do not arise from lack of convergence in the FSSH simulations. The physical meaning of such oscillations, if any, perhaps can be further elucidated through the use of full quantum dynamical simulations that treat the nuclear degrees of freedom on equal quantum mechanical footing as the electronic and photonic degrees of freedom. In the moderate loss regime where there is still significant coupling between the $|L P\rangle$ and $|U P\rangle$ states coincident with dissipative features, it is observed that cis-to-trans isomerization is strongly suppressed as relaxation to the $|g, 0\rangle$ state competes with motion along the isomerization coordinate (see Figure 5). Investigation of the time-dependent transition probabilities around the avoided crossing (when $R=-0.6$ a.u.) reveals that a $|U P\rangle \rightarrow|L P\rangle \rightarrow|g, 0\rangle$ relaxation channel is uniquely facile under moderate loss conditions (see Figure S6 in the Supporting Information).

We have developed a non-Hermitian formulation of polaritonic structure modeled after the paradigmatic system of azobenzene coupled to a cavity that explicitly accounts for cavity losses. We include only the isomerization coordinate (what would correspond to the $\mathrm{C}-\mathrm{N}-\mathrm{N}-\mathrm{C}$ dihedral angle in azobenzene) in our simplified model. That said, a non-Hermitian formula- 


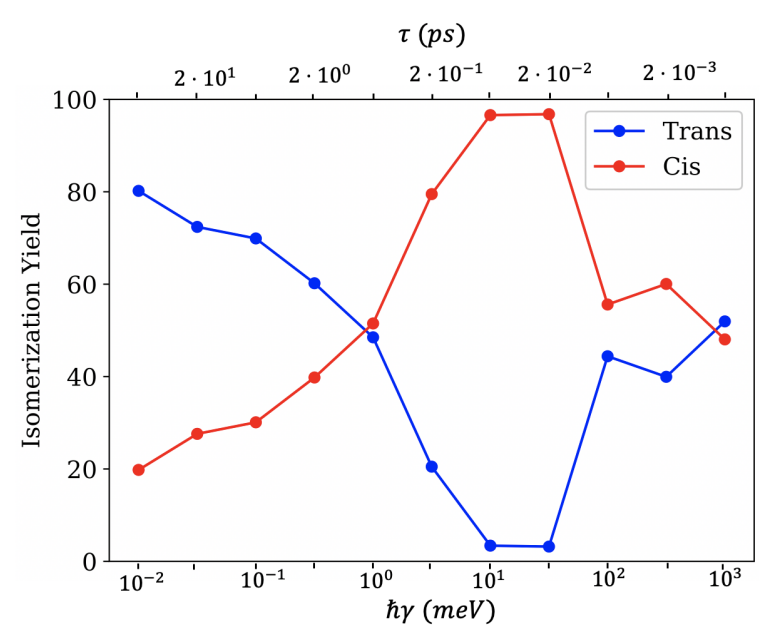

Figure 5: The isomerization yield for surface hopping trajectories initialized in the cis- configuration on the $|U P\rangle$ surface. The isomerization yield of the transisomer goes to zero for moderate dissipation rates, but at high dissipation rates, the isomerization yield approaches $50 \%$ cis- and $50 \%$ trans, similar to the isomerization of lone azobenzene.

tion of polaritonic structure that includes multiple modes and/or an ab initio treatment of the molecular degrees of freedom is certainly possible and will be pursued in future work.

Our approach demonstrates that several features of the polaritonic structure are altered by the dissipative features of the cavity, and that these modifications can have profound impacts on the isomerization dynamics in this system. In particular, we find that cavityenhanced isomerization reported in prior works is maintained only when photonic dissipation rates are $\leq 1 \mathrm{meV}$, though this does not necessarily preclude favorable balances between coupling and dissipation that could allow polaritonic chemical control with larger photonic dissipation rates. Isomerization is strongly suppressed for moderate dissipation rates, and in the limit of large cavity dissipation, the isomerization dynamics resemble that of the cavityfree system. These results suggest that explicit inclusion of cavity losses are critical for simulating polaritonic chemistry, and also points to the importance of loss engineering in optical cavities for polaritonic chemistry applications.

Acknowledgement This work was performed, in part, utilizing resources at the
Center for Nanoscale Materials, a US Department of Energy,Office of Science, Office of Basic Energy Sciences User Facility (contract no. DE-AC02-06CH11357). High-performance computing resources of the MERCURY Consortium (http://www.mercuryconsortium.org) were used. JJF and FS acknowledge the Research Corporation for Scientific Advancement Cottrell Scholar Award for support. JJF, JFV and PA acknowledge the donors of the American Chemical Society Petroleum Research Fund for partial support of this work. JJF acknowledges the ART program at William Paterson University for partial support of this work.

Supporting Information Available: Supporting figures, further technical details on the theoretical methodology, and simulation details are provided in the Supporting Information.

\section{References}

(1) Bellessa, J.; Bonnand, C.; Plenet, J. C.; Mugnier, J. Strong coupling between surface plasmons and excitons in an organic semiconductor. Phys. Rev. Lett 2004, 93, 036404 .

(2) Hutchison, J. A.; Schwartz, T.; Genet, C.; Devaux, E.; Ebbesen, T. W. Modifying chemical landscapes by coupling to vacuum fields. Angew. Chem. Int. Ed. 2012, 51, 1592-1596.

(3) Schwartz, T.; Hutchison., J.; Leonard, J.; Genet, C.; Haacke, S.; Ebbesen, T. Polariton dynamics under strong light-molecule coupling. Chem. Phys. Chem 2013, 14, 125-131.

(4) Galego, J.; Garcia-Vidal, F. J.; Feist, J. Cavity-induced modifications of molecular structure in the strong-coupling regime. Phys. Rev. X 2015, 5, 041022.

(5) Ebbesen, T. W. Hybrid light-matter states in a molecular and material science perspective. Acc. Chem. Res. 2016, 49, 24032412. 
(6) Thomas, R.; Thomas, A.; Pullanchery, S.; Joseph, L.; Somasundaran, S. M.; Swathi, R. S.; Gray, S. K.; Thomas, K. G. Plexitons: The role of oscillator strengths and spectral widths in determining strong coupling. ACS Nano 12, 402-415.

(7) Hoffmann, N. M.; Appel, H.; Rubio, A.; Maitra, N. T. Light-matter interactions via the exact factorization approach. Eur. Phys. J. B 2018, 91, 180.

(8) Mandal, A.; Huo, P. Investigating new reactivities enabled by polariton photochemistry. J. Phys. Chem. Lett. 2019, 10, 5519-5529.

(9) Du, M.; Ribeiro, R. F.; Yuen-Zhou, J. Remote control of chemistry in optical devices. Chem. Cell. Press 2019, 5, 11671181.

(10) Eldabagh, N.; Micek, M.; III, A. E. D. Resonance energy transfer mediated by metal-dielectric composite nanostructures. J. Phys. Chem. C 2018, 122, 18256-18265.

(11) Herrera, F.; Spano, F. C. Cavitycontrolled chemistry in molecular ensembles. Phys. Rev. Lett. 2016, 116, 238301.

(12) Flick, J.; Ruggenthaler, M.; Appel, H.; Rubio, A. Atoms and molecules in cavities, from weak to strong coupling in quantum-electrodynamics (QED) chemistry. Proc. Natl. Acad. Sci. USA 2017, 114, 3026-3034.

(13) Galego, J.; Garcia-Vidal, F. J.; Feist, J. Many-molecule reaction triggered by a single photon in polaritonic chemistry. Phys. Rev. Lett. 2017, 119, 136001.

(14) Flick, J.; Appel, H.; Ruggenthaler, M.; Rubio, A. Cavity Born-Oppenheimer approximation for correlated electronnuclear-photon systems. J. Chem. Theor. Comput. 2017, 13, 1616-1625.
(15) Martínez-Martínez, L. A.; Ribeiro, R. F.; Campos-Gonzalez-Angulo, J.; YuenZhou, J. Can ultrastrong coupling change ground-state chemical reactions? ACS Photonics 2018, 5, 167-176.

(16) Martínez-Martínez, L. A.; Du, M.; Ribeiro, R. F.; Kéna-Cohen, S.; YuenZhou, J. Polariton-assisted singlet fission in acene aggregates. J. Phys. Chem. Lett. 2018, 9, 19511957.

(17) Fregoni, J.; Granucci, G.; Coccia, E.; Persico, M.; Corni, S. Manipulating azobenzene photoisomerization through strong lightmolecule coupling. Nat. Commun. 2018, 9, 4688.

(18) Martínez-Martínez, L. A.; Eizner, E.; Kéna-Cohen, S.; Yuen-Zhou, J. Triplet harvesting in the polaritonic regime: A variational polaron approach. J. Chem. Phys. 2019, 151, 054106.

(19) Zhang, Y.; Nelson, T.; Tretiak, S. Nonadiabatic molecular dynamics of molecules in the presence of strong light-matter interactions. J. Chem. Phys. 2019, 151, 154109 .

(20) Fregoni, J.; Granucci, G.; Persico, M.; Corni, S. Strong coupling with light enhances the photoisomerization quantum yield of azobenzene. Chem 2020, 6, 250265.

(21) Fregoni, J.; Corni, S.; Persico, M.; Granucci, G. Photochemistry in the strong coupling regime: A trajectory surface hopping scheme. J. Comput. Chem. 2020, 41, 2033-2044.

(22) Felicetti, S.; Fregoni, J.; Schnappinger, T.; Reiter, S.; de Vivie-Riedle, R.; Feist, J. Photoprotecting uracil by coupling with lossy nanocavities. J. Phys. Chem. Lett. 2020, 11, 8810-8818.

(23) Ulusoy, I. S.; Vendrell, O. Dynamics and spectroscopy of molecular ensembles in a lossy microcavity. J. Chem. Phys. 2020, 153, 044108. 
(24) Bandara, H. M. D.; Burdette, S. C. Photoisomerization in different classes of azobenzene. Chem. Soc. Rev 2012, 41, 1809-1825.

(25) Baroncini, M.; Groppi, J.; Corra, S.; Silvi, S.; Credi, A. Light-responsive (supra)molecular architectures: Recent advances. Adv. Opt. Mat 2019, \%, 1900392.

(26) Hoffman, N. M.; Schäfer, C.; Sä, N.; Rubio, A.; Appel, H.; Kelly, A. Benchmarking semiclassical and perturbative methods for real-time simulations of cavitybound emission and interference. J. Chem. Phys. 2019, 151, 244113.

(27) Tokatly, I. V. Time-dependent density functional theory for many-electron systems interacting with cavity photons. Phys. Rev. Lett. 2013, 110, 23301.

(28) Flick, J.; Narang, P. Cavity-correlated electron-nuclear dynamics from first principles. Phys. Rev. Lett. 2018, 121, 113002.

(29) Buchholz, F.; Theophilou, I.; Nielsen, S. E. B.; Ruggenthaler, M.; Rubio, A. Reduced density-matrix approach to strong matter-photon interaction. ACS Photonics 2019, 6, 2694-2711.

(30) Mordovina, U.; Bungey, C.; Appel, K.; Knowles, P. J.; Rubio, A.; Manby, F. R. Polaritonic coupled-cluster theory. Phys. Rev. Research 2020, 2, 023262.

(31) Rivera, N.; Flick, J.; Narang, P. Variational theory of nonrelativistic quantum electrodynamics. Phys. Rev. Lett. 2019, 122, 193603.

(32) Wang, D. S.; Neuman, T.; Flick, J.; Narang, P. Weak-to-strong light-matter coupling and dissipative dynamics from first principles. arXiv 2020, 2002, 10461v1.

(33) Cortes, C. L.; Otten, M.; Gray, S. K. Non-Hermitian approach for quantum plasmonics. J. Chem. Phys. 2020, 152, 084105.
(34) Tully, J. C. Molecular dynamics with electronic transitions. J. Chem. Phys. 1990, 93, 1061.

(35) Subotnik, J. E.; Jain, A.; Landry, B.; Petit, A.; Ouyang, W.; Bellonzi, N. Understanding the surface hopping view of electronic transitions and decoherence. Annu. Rev. Phys. Chem. 2016, 67, 387417.

(36) Tempelaar, R.; van der Vegte, C. P.; Knoester, J.; Jansena, T. L. C. Surface hopping modeling of two-dimensional spectra. J. Chem. Phys. 2013, 138, 164106.

(37) Kossoski, F.; Barbatti, M. Nonadiabatic dynamics in multidimensional complex potential energy surfaces. Chem. Sci. 2020 ,

(38) Medina, I.; Garca-Vidal, F. J.; FernndezDomnguez, A. I.; Feist, J. Few-mode Field Quantization of Arbitrary Electromagnetic Spectral Densities. arXiv 2020, 2008, 00349v1.

(39) Hoffman, N. M.; Lacombe, L.; Rubio, A.; Maitra, N. T. Effect of many modes on self-polaritzation and photochemical suppression in cavities. J. Chem. Phys. 2020, 153, 104103.

(40) Mandal, A.; Krauss, T. D.; Huo, P. Polariton-mediated electron transfer via cavity quantum electrodynamics. J. Phys. Chem. B 2020, 124, 6321-6340.

(41) Taylor, M. A. D.; Mandal, A.; Zhou, W.; Huo, P. Resolution of gauge ambiguities in molecular cavity quantum electrodynamics. Phys. Rev. Lett. 2020,

(42) Stefano, O. D.; Settineri, A.; Macri, V.; Garziano, L.; Stassi, R.; Savasta, S.; Nori, F. Resolution of gauge ambiguities in ultrastrong-coupling cavity quantum electrodynamics. Nat. Phys. 2019, 15,803808 .

(43) Schäfer, C.; Ruggenthaler, M.; Rokaj, V.; Rubio, A. Relevance of the quadratic 
diamagnetic and self-polarization terms in cavity quantum electrodynamics. ACS Photonics 2020, 7, 975-990.

(44) Satzger, H.; Spörlein, S.; Root, C.; Wachtveitl, J.; Zinth, W.; Gilch, P. Fluorescence spectra of trans- and cisazobenzene-emission from the FranckCondon state. Chem. Phys. Lett. 2003, 372, 216-223.

(45) Jaynes, E. T.; Cummings, F. W. Comparison of quantum and semiclassical radiation theories with application to the beam maser. Proc. IEEE 1963, 51, 89-109.

(46) Pan, F.; Smith, K. C.; Nguyen, H. L.; Knapper, K. A.; Masiello, D. J.; Goldsmith, R. H. Elucidating energy pathways through simultaneous measurement of absorption and transmission in a coupled plasmonic-photonic cavity. Nano Lett. 2020, 20, 50-58.

(47) Smith, H. T.; Karam, T. E.; Haber, L. H.; Lopata, K. Capturing plasmon-molecule dynamics in dye monolayers on metal nanoparticles using classical electrodynamics with quantum embedding. J. Phys. Chem. C 2017, 121, 16932-16942.

(48) Nascimento, D. R.; DePrince III, A. E. Modeling molecule-plasmon interactions using quantized radiation fields within time-dependent electronic structure theory. J. Chem. Phys. 2015, 143, 214104. 